\title{
A predictive tool for foreign body fibrotic reactions using a two-dimensional computational model
}

This article was published in the following Dove Press journal:

Open Access Bioinformatics

18 January 201 I

Number of times this article has been viewed

\author{
Jianzhong Su' \\ Michail Todorov 1,3 \\ Humberto Perez Gonzales' \\ Larrissa Perkins' \\ Hristo Kojouharov' \\ Hong Weng ${ }^{2}$ \\ Liping Tang ${ }^{2}$ \\ 'Department of Mathematics, \\ ${ }^{2}$ Department of Bioengineering, \\ University of Texas at Arlington, \\ Arlington, TX, USA; ${ }^{3}$ Faculty of \\ Applied Mathematics and Informatics, \\ Technical University of Sofia, Sofia, \\ Bulgaria
}

\begin{abstract}
It is well established that implanted medical devices often trigger immunological and inflammatory reactions. Such foreign body-mediated tissue responses may result in fibrotic tissue formation surrounding the implants. Despite intensive research in the area of wound healing, few methods are currently available to systematically predict the quantitative behavior of this complex system of multiple cells, proteins, and enzymes. This study introduces a kinetics-based predictive tool in order to analyze outcomes of reactions of various cells/proteins and biochemical processes and to understand transient behavior during the entire implant healing period of up to several months. A computational model in two spatial dimensions is constructed to investigate the time dynamics as well as spatial variation of fibrotic reaction kinetics. Our results support that this model can be used to predict many features in a systematic way and also to forecast the outcomes of complex tissue responses to biomaterial implants.
\end{abstract}

Keywords: mathematical model, inflammatory responses, foreign body reactions, fibrosis, predictive tool

\section{Introduction}

All medical implants prompt varying degrees of inflammatory tissue reactions, which may in severe cases lead to the failure of medical devices. ${ }^{1}$ Although the sequence of events leading to fibrosis can be complex or even convoluted, it is generally agreed that such foreign body reactions are initiated by implant-mediated fibrin clot formation followed by acute inflammatory responses. ${ }^{1,2}$ The inflammatory chemokines released by adherent immune cells serve as strong signals for triggering the migration of macrophages and fibroblasts toward the implant surface from the surrounding tissues and circulation. ${ }^{3}$ The implant-recruited fibroblasts consequently synthesize chains of amino acids called procollagens, a process that is activated by growth factors, including, in particular, transforming growth factor type $\beta$ (TGF- $\beta$ ). ${ }^{4,5}$ The procollagens then get converted by enzymes ${ }^{6}$ into collagens, the dominant ingredient of the extracellular matrix (ECM). Many cells and proteins participate in the process and form a network of signaling pathways that, in turn, lead to inflammatory and fibrotic reactions. For example, inactive (latent forms of) TGF- $\beta$ isoforms are also secreted by many cells, ${ }^{7,8}$ and they have a much slower decay rate than their respective active TGF- $\beta$ forms. ${ }^{9}$ Once they are activated by specific enzymes, the active TGF- $\beta$ will then initiate the growth of fibroblasts and consequently secrete procollagens. The implant site contains enzymes that activate latent growth factors and stabilize collagen structure. ${ }^{10}$ Similar to other collagen formation processes such as dermal wound healing, inactive collagenase (called zymogen) is synthesized and secreted by fibroblast cells, and once
Correspondence: Jianzhong Su

Department of Mathematics, University of Texas at Arlington, Arlington, TX

76019 , USA

Tel +l 8172725684

Fax +l 8172725802

Email su@uta.edu 
the zymogen is activated to active collagenase, collagen degradation occurs at the same time as collagen formation. ${ }^{11}$ Among inflammatory cells, macrophages (MФ) are found to reside in the wound and to exert long-term tissue reactions. ${ }^{1,12}$ Specifically, МФ remove damaged tissue and foreign debris via phagocytosis. In addition, $\mathrm{M} \Phi$ may release a variety of chemokines to recruit other cell types, such as fibroblasts, which participate in the remodeling of the ECM. ${ }^{13} \mathrm{M} \Phi$ are classified into three phenotypes according to their roles in the wound-healing process. First, classically activated $\mathrm{M} \Phi$ designate the effector $\mathrm{M} \Phi$ that are produced during cellmediated immune responses. Two signals, interferon- $\gamma$ and tumor-necrosis factor- $\alpha$, result in the effector $М \Phi$, have enhanced microbicidal or tumoricidal capacity, and secrete high levels of proinflammatory cytokines and mediators. Second, the clearance of apoptotic inflammatory as well as noninflammatory cells by $\mathrm{M} \Phi$, including polymorphonuclear cells, can lead to an inhibition of inflammation, owing in part to the production of TGF- $\beta .{ }^{14,15}$ Wound-healing MФ can develop in response to innate or adaptive signals through interleukin 4. In turn, interleukin 4 stimulates arginase activity in $\mathrm{M} \Phi$, allowing them to convert arginine to ornithine, a precursor of polyamines and collagen, thereby contributing to the production of ECM. ${ }^{16}$ Finally, regulatory $М \Phi$ can also arise during the later stages of adaptive immune responses, the primary role of which is to dampen the immune response and limit inflammation through production of interleukin $10 .{ }^{17}$ Although all three types are observed experimentally in the dermal wound-healing process, the phagocytes biomaterial interactions are known to be similar. ${ }^{2}$

Recent research efforts have been focused on developing mechanistic models for the wound-healing process using system biology methodology. Significant progress has been made in the area of wound healing where the predictive models, based on biochemical and biophysics mechanisms, have been used for analysis of treatments. ${ }^{18}$ Wound-healing responses and foreign body reactions involve the same groups of biochemical agents. However, these processes may differ in specific activation and inhibition loops. In the pursuit of a greater understanding of these foreign body reactions, computational models can be used to systematically study the complex immune processes that are involved, complementing experimental work. Our primary goal in this article is to model, using computational tools, the fibrotic reaction process following implantation. Such a quantitative study is useful for investigating the possible pathways of reaction networks by including various tissue growth factors, enzymes, and cells, as well as their migrations that redistribute in space via diffusions, through a continuum model or a continuous-discrete multiscale model. Furthermore, because of the importance of implant surface properties to foreign body reactions, numerical simulations are performed to test biological hypotheses on reactions with chemical-coated biomaterial surfaces, as described earlier. ${ }^{19}$ Our modeling results indicate trends for these variations, serving as a plausible clue for developing new experiments.

\section{Materials and methods Modeling considerations}

The process of foreign body inflammatory reactions to implants involves complex interactions of many types of cells and proteins and occurs as a cascade of sequential, parallel, and overlapping chemical processes. ${ }^{20}$ During a short initiation period of surface-mediated reactions, mainly involving implant-mediated fibrin clot formation followed by acute inflammatory responses, various tissue growth factors are released. ${ }^{1,21}$ In response to the gradient field of tissue growth factors released in the implant domain, fibroblasts migrate toward the implant from the surrounding healthy tissues and/ or proliferate within scaffoldings, which mainly consist of fibrinogen layers that are absorbed on the implant's surface. The gradient-sensing mechanism can be captured in essence by either chemotactic equations in continuum models or cell motion equations of discrete cells (multiscale models). Upon reaching the implantation site, recruited cells such as МФ and fibroblasts continue to release more chemotactic agents ${ }^{22}$ to reinforce the gradient fields and to recruit more cells. The continuous cell recruitment can be modeled by chemotactic equations. $^{22}$ The fibroblasts consequently synthesize procollagens, which get converted into collagens. ${ }^{4}$ These chemical reactions, however, involve many other ingredients such as both active and latent forms of TGF- $\beta$ isoforms secreted by many cells. ${ }^{7-9}$ The implant site contains enzymes that activate latent growth factors and stabilize collagen formations. ${ }^{10}$ On the other hand, collagen formations are also partially reversible and subject to natural degradation. Consistent with collagen formation in dermal wound healing, zymogen is synthesized and secreted by fibroblasts, ${ }^{11}$ and once the zymogen is activated by enzymes to become collagenase, collagen degradation and collagen formation occur concurrently.

The basic reactions were considered in previous modeling studies, ${ }^{23,24}$ and some of their corresponding key features of kinetics were incorporated in our modeling. We refer interested readers to Dallon et $\mathrm{al}^{25}$ for a survey of the field as well as more thorough bibliographical references. Our model will further 
include several features of $\mathrm{M} \Phi$ in the implantation process, as they play a unique role in foreign body reactions. At the implant domain, a small number of $\mathrm{M} \Phi$ ignite the cascades of immune responses. The number and density of M $\Phi$ cells do not increase significantly with time. We thus treated $M \Phi$ as either a continuum or as discrete cells in two different models in our study. Although the discrete cell modeling is capable of showing very interesting development patterns of the healing process, continuum modeling provides a good estimation of average behavior. The model also reflects some features such as cell apoptosis and clearance of apoptotic cells, which can lead to an inhibition of inflammation. ${ }^{14} \mathrm{M} \Phi$ have been shown to have multiple roles, as described in Mosser and Edwards, ${ }^{13}$ and all three phenotypes are observed experimentally, but in order to avoid being too overly specific in modeling these processes, we summarize these reactions as part of a specific enzyme reaction to account for these changes.

\section{Method of mathematical modeling based on chemical kinetics equations}

Our modeling work starts with the mass action kinetics framework developed by Dale et al, ${ }^{23,26}$ which was developed to simulate normal adult and fetal wound healing. To better depict foreign body reactions, new kinetics of $М \Phi$ reactions were added in the framework. The foreign body reaction dynamics also reside in a different parametric range from the standard model in Dale et al, ${ }^{23}$ which gave different dynamic characters. The main biological difference between these two responses is that foreign body reactions are deep subdermal phenomena, and normal wound-healing processes are based on superficial dermal wound experimental data.

Table I Variables in foreign body reactions (after scaling, variables are dimensionless)

\begin{tabular}{ll}
\hline$f(x, y, t)$ & Fibroblast density \\
$\beta_{1}(x, y, t)$ & Total active TGF- $\beta$ isoform I and isoform \\
& 2 density \\
$\beta_{3}(x, y, t)$ & Active TGF- $\beta$ isoform 3 density \\
$I_{1}(x, y, t)$ & Total latent TGF- $\beta$ isoform I and isoform \\
& 2 density \\
$I_{3}(x, y, t)$ & Latent TGF- $\beta$ isoform 3 density \\
$e_{1}(x, y, t), e_{2}(x, y, t), e_{3}(x, y, t)$ & Generic enzymes type I, II, III densities \\
$p_{1}(x, y, t), p_{3}(x, y, t)$ & Procollagens (latent collagens) I and III \\
$c_{1}(x, y, t), c_{3}(x, y, t)$ & densities \\
$z_{1}(x, y, t), z_{3}(x, y, t)$ & Collagens I and III densities \\
$s_{1}(x, y, t), s_{3}(x, y, t)$ & Zymogens (latent collagenases) I and III \\
$I(x, y, t)$ & densities \\
\hline
\end{tabular}

The variables considered in the modeling are summarized in Table 1.

The spatial variables $x$ and $y$ (unit: $\mathrm{cm}$ ) denote the location in the reaction domain, immediately between the implant and normal healthy tissues, where foreign body reactions occur. Specific geometry of the implant and reaction domain is provided in spatial patterns of collagen growth that form a propagating wave toward the implant. The variable $t$ (unit: day) is the time. Fibroblast density, $f(x, y, t)$, represents the main cell type in the implant domain. We ignore directional effects of fibroblast cells and assume that cell migration is through diffusion. Fibroblast proliferation and collagen synthesis are upregulated by active forms of TGF- $\beta s .{ }^{27}$ The effect of various isoforms of TGF- $\beta, \beta_{1}(x, y, t)$ and $\beta_{3}(x, y, t)$, on the cell fibroblast population can be approximated by a chemically enhanced logistic growth $\left(A_{1}+A_{2} \beta_{1}+A_{3} \beta_{3}\right) f\left(1-f / k_{1}\right)$ with a threshold $k_{1}$, along with its diffusion in space modeled by $D_{1} \nabla^{2} f$ and its natural decay according to time $-A_{4} f$ :

$$
\frac{\partial f}{\partial t}=D_{1} \nabla^{2} f+\left(A_{1}+A_{2} \beta_{1}+A_{3} \beta_{3}\right) f\left(1-\frac{f}{k_{1}}\right)-A_{4} f .
$$

Latent TGF- $\beta$ isoforms $l_{1}(x, y, t)$ and $l_{3}(x, y, t)$ that diffuse in space modeled by $\nabla^{2} l_{1}$ and $\nabla^{2} l_{3}$ are produced by fibroblasts via autocrine regulation in amounts $A_{5} f l_{1} /\left(1+A_{6} l_{3}+A_{7} l_{1}\right)$ and $A_{9} f l_{3} /\left(1+A_{10} l_{3}\right)$, respectively. ${ }^{28}$ On the other hand, latent TGF- $\beta$ has natural decays as well as loss due to activations $l_{1}(x, y, t)$ and $l_{3}(x, y, t)$ to $\beta_{1}(x, y, t)$ and $\beta_{3}(x, y, t)$ by specific enzyme $e_{1}$, as reflected by $-A_{16} e_{1} l_{1}$ and $-A_{17} e_{1} l_{3}$ in Eqs 2 and 3 . These converted active TGF- $\beta$ s are then reflected in Eqs 4 and 5 as $A_{12} e_{1} l_{1}$ and $A_{14} e_{1} l_{3}$, respectively. These active forms of TGF- $\beta$ undergo similar diffusions and natural decays. We assume all forms of TGF- $\beta$ s have constant diffusion coefficients. The TGF- $\beta$ s are modeled by equations:

$$
\begin{gathered}
\frac{\partial l_{1}}{\partial t}=D_{2} \nabla^{2} l_{1}+\frac{A_{5} f l_{1}}{1+A_{6} l_{3}+A_{7} l_{1}}-A_{8} l_{1}-A_{16} e_{1} l_{1}, \\
\frac{\partial l_{3}}{\partial t}=D_{3} \nabla^{2} l_{3}+\frac{A_{9} f l_{3}}{1+A_{10} l_{3}}-A_{11} l_{3}-A_{17} e_{1} l_{3}, \\
\frac{\partial \beta_{1}}{\partial t}=D_{4} \nabla^{2} \beta_{1}+A_{12} e_{1} l_{1}-A_{13} \beta_{1}, \\
\frac{\partial \beta_{3}}{\partial t}=D_{5} \nabla^{2} \beta_{3}+A_{14} e_{1} l_{3}-A_{15} \beta_{3} .
\end{gathered}
$$

During early stages of foreign body implantation, monocytes and $М \Phi$ release a range of enzymes, which convert latent 
growth factors, procollagens, and zymogens into their respective active forms. ${ }^{29}$ We use the law of mass action to model activations of latent TGF- $\beta 1$ and 3, latent collagen I and III, and latent collagenases I and III, by $e_{1}, e_{2}$, and $e_{3}$, respectively, in Eqs 6-8. Although $\mathrm{M} \Phi$ secrete all three enzymes, the effect on enzyme $e_{2}$ has been most significant. We model additional secretion in $e_{2}$ from M $\Phi$ in an additional amount of $B_{36} l$ :

$$
\begin{gathered}
\frac{\mathrm{d} e_{1}}{\mathrm{dt}}=-e_{1}\left(A_{16} l_{1}+A_{17} l_{3}\right), \\
\frac{\mathrm{d} e_{2}}{\mathrm{dt}}=-e_{2}\left(A_{18} p_{1}+A_{19} p_{3}\right)+B_{36} l, \\
\frac{\mathrm{d} e_{3}}{\mathrm{dt}}=-e_{3}\left(A_{40} z_{1}+A_{41} z_{3}\right) .
\end{gathered}
$$

Fibroblasts secrete procollagens, ${ }^{4}$ and the production is promoted by active TGF- $\beta$. In Eqs 9 and 10, procollagens are synthesized by fibroblasts in the amounts of $\left(A_{20}+A_{21} \beta_{1}+A_{22} \beta_{3}\right) f$ and $\left(A_{24}+A_{25} \beta_{1}+A_{26} \beta_{3}\right) f$, whereas their concentration is decreased due to activation to the collagens by specific enzyme $e_{2}$ in amounts of $-A_{18} e_{2} p_{1}$ and $-A_{19} e_{2} p_{3}$, respectively. The amounts of activated procollagen are added to the collagen equations (Eqs 11 and 12), which are balanced by the degradations through their collagenases in amounts of $-A_{29} s_{1} c_{1}$ and $-A_{31} s_{3} c_{3}$, respectively.

$$
\begin{aligned}
& \frac{\mathrm{d} p_{1}}{\mathrm{dt}}=\left(A_{20}+A_{21} \beta_{1}+A_{22} \beta_{3}\right) f-A_{23} p_{1}-A_{18} e_{2} p_{1}, \\
& \frac{\mathrm{d} p_{3}}{\mathrm{dt}}=\left(A_{24}+A_{25} \beta_{1}+A_{26} \beta_{3}\right) f-A_{27} p_{3}-A_{19} e_{2} p_{3}, \\
& \frac{\mathrm{d} c_{1}}{\mathrm{dt}}=A_{28} p_{1} e_{2}-A_{29} S_{1} c_{1}, \\
& \frac{\mathrm{d} c_{3}}{\mathrm{dt}}=A_{30} p_{3} e_{2}-A_{31} s_{3} c_{3} \text {. }
\end{aligned}
$$

We model processes of collagenases in Eqs 13 and 14 and latent forms of collagenases in Eqs 15 and 16 based on the same mass action laws established earlier, and we refer readers to Dale et $\mathrm{al}^{23}$ for more explanations of mass action laws. The conversion is by specific enzyme $e_{3}$ and $\mathrm{M} \Phi$ secrete collagenases $B_{37} l$ and $B_{38} l$. We summarize laws of both forms of collagenases as

$$
\begin{aligned}
\frac{\mathrm{d} z_{1}}{\mathrm{dt}} & =\frac{A_{32}}{1+A_{33} \beta_{1}+A_{34} \beta_{3}} f c_{1}-A_{35} z_{1}-A_{40} e_{3} z_{1}+B_{37} l, \\
\frac{\mathrm{d} z_{3}}{\mathrm{dt}} & =\frac{A_{36}}{1+A_{37} \beta_{1}+A_{38} \beta_{3}} f c_{3}-A_{39} z_{3}-A_{41} e_{3} z_{3}+B_{38} l
\end{aligned}
$$

$$
\begin{gathered}
\frac{\mathrm{d} s_{1}}{\mathrm{dt}}=A_{42} z_{1} e_{3}-A_{43} s_{1}, \\
\frac{\mathrm{d} s_{3}}{\mathrm{dt}}=A_{44} z_{3} e_{3}-A_{45} s_{3} .
\end{gathered}
$$

Inflammatory cells, $\mathrm{M} \Phi$, behave differently at different stages of foreign body reactions. Our model differs from early collagen models primarily because we incorporate the following features: a) M $\Phi$ produce procollagen specific enzymes at a near-saturated level and b) $M \Phi$ regulate collagen growth through production of zymogens. These changes led to substantial quantitative behavior changes in the model. The activation and proliferation of $M \Phi$ are through upregulation of growth factors TGF- $\beta \mathrm{s},{ }^{30}$ but the production does reach a limiting value once TGF- $\beta$ s reach saturation, and this can be modeled by $B_{39}\left(e_{1} l_{1}+e_{1} l_{3}\right) l\left(\left(N_{n}+N_{e}\right) /\left(N_{\mathrm{n}}+\right.\right.$ l)). Programmed death (apoptosis) of M $\Phi$ normally occurs after several weeks to a month and is modeled by $-B_{41} \sigma_{0}(t-$ $30) l$, where a life span of 30 days is assumed. The degree of macrophage response is modeled as

$$
\begin{aligned}
\frac{\mathrm{d} l}{\mathrm{dt}}= & D_{6} \nabla^{2} l+B_{39}\left(e_{1} l_{1}+e_{1} l_{3}\right) l \frac{\left(N_{n}+N_{e}\right)}{N_{n}+l} \\
& -B_{40} l-B_{41} \sigma_{0}(t-30) l .
\end{aligned}
$$

\section{Experimental validation}

Experiments on collagen growth were performed using a mouse subcutaneous implantation model as described earlier. ${ }^{31}$ Briefly, disks (1.2 cm diameter) of polyethylene terephthalate (PET, Cadillac Plastic and Chemical Company, Birmingham, MI, USA) were sterilized with multiple washes of $70 \%$ ethanol. PET disks were implanted subcutaneously in the dorsal area on the back of mice. The small incision was then closed with stainless steel wound clips. After implantation for different periods of time $(4,7,14,21$, and 28 days), the mice were sacrificed, and the implants were then recovered for collagen measurement. We measured the amount of hydroxyproline that makes up $12 \%-14 \%$ of type I collagen, which, in turn, constitutes roughly $80 \%$ of the total collagen. Hydroxyproline was measured by established techniques. ${ }^{32}$ Our recent results have shown that increasing amounts of collagen were produced by adherent fibroblasts with time and achieved plateau around 21-28 days (Table 2). The experimental results were then compared with the simulation results from our model. The quality was rescaled before comparison, because the experiments are based on 
Table 2 Experimental data of collagen deposit in a polyethylene terephthalate (PET) membrane implanted in mice for different periods of time

\begin{tabular}{|c|c|c|c|c|c|}
\hline \multicolumn{6}{|c|}{ Collagen deposition on PET $20-\mu \mathrm{m}$ membrane $\left(\mu \mathrm{g} / \mathrm{cm}^{2}\right)$} \\
\hline & $\mathbf{I}$ & 2 & 3 & 4 & 5 \\
\hline 4 days & 1.5 & 1.12 & 0.847 & 0.957 & 0.957 \\
\hline 7 days & 19.63 & 19.12 & 20.29 & 25.8 & 27.43 \\
\hline 14 days & 149.96 & 74.73 & 89.54 & 128.82 & 110.76 \\
\hline 2I days & 106.32 & 115.52 & 140.52 & 132.94 & 115.89 \\
\hline 28 days & I48.32 & 134.92 & 179.93 & 111.8 & | 48.34 \\
\hline
\end{tabular}

surface density and our model works on volume density. We demonstrate in Figure 1 the comparison of the three data sets - experimental, the Dale computational model, and our computational model - for the set of parameters given above. These preliminary results were obtained in our studies. ${ }^{33,34}$

Using the same set of parameter values, we compared the experimental results with simulation outcomes based on Dale's model as well as our improved model (Figure 1). Although the Dale model was successful in modeling wound healing, it cannot be applied directly to foreign body reaction problems because of its limitations. Especially, Dale's model cannot be used to simulate the plateau of collagen production. Thus, there is an essential need for modeling of $\mathrm{M} \Phi$ and incorporation into the foreign body reactions model. As discussed in Modeling considerations, our important modification to the classical Dale model is to incorporate the feature of MФ kinetics in the reactions. From a physiological point of view, $\mathrm{M} \Phi$ and subsequent foreign body reaction giant cells (ie, fused M $\Phi$ cells) are the main driving force behind the cascades of immunological responses. From a quantitative

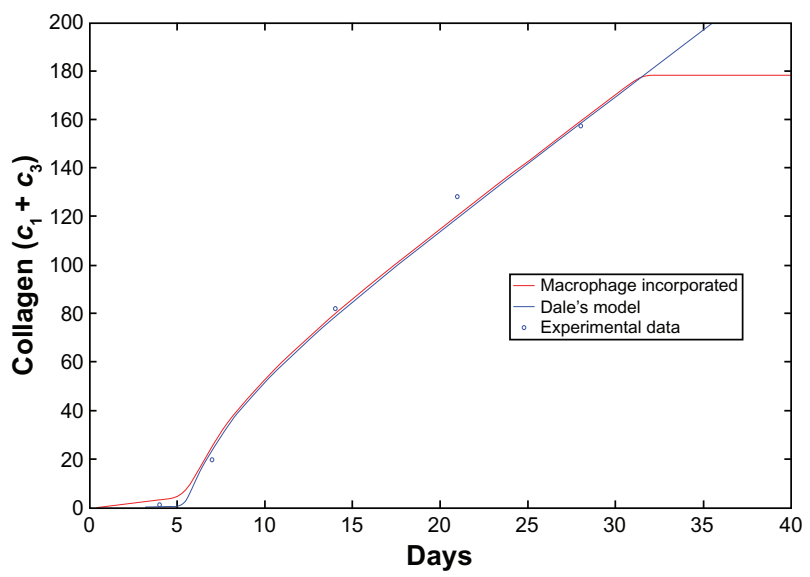

Figure I The comparison of collagen level in experimental data (the average value of samples during a 28-day period, $\mu \mathrm{g} / \mathrm{cm}^{2}$ ) with simulated data from the mathematical models. The classical Dale model has an asymptotic collagen variable that increases linearly as time increases. Our improved foreign body reaction model incorporated $M \Phi$ cell using physiological principle and mass action law, and the modified mode (red) is shown to have a realistic behavior. point of view, these two models also have a clear distinction. In terms of the spatial variation, $\mathrm{M} \Phi$ cells lead the way in implant association, and their gradient field further attracts other cells to the site. Differing from wound healing, where collagen or scar formation occurs after several days, the foreign body reactions are slow developing processes that take up to several months, albeit the ingredients may react in several time scales (days, weeks, or months).

\section{Continuous-discrete modeling methods}

Unlike battlefield injury, burns, and other wound-healing processes, the medical implantation process is a controlled procedure in which infections are carefully avoided. It is also a reasonable assumption that $\mathrm{M} \Phi$ are relatively small in numbers, to a degree that a continuum model may stretch their limitations. We therefore built a multiscale model that combines continuous modeling for the cellular and chemical fields along with discrete modeling for limited numbers of $M \Phi$ For our continuous-discrete foreign body reaction model, we made the following assumptions: 1) the number of MФ cells is finite, and these discretely distributed cells observe chemotactic law; and 2) fibroblasts and other elements satisfy continuum mass action law, and their continuum fields are influenced by discrete $\mathrm{M} \Phi$ cells. To simplify the model, we hold that a MФ is a volumeless point but has an effective radius of influence through the release of various enzymes and proteins, and the rate of releasing enzymes and proteins is proportional to a Gaussian 'bell'-shaped weight function of distance, measuring from the field location to the location of the said MФ cell.

The new discrete model replaces Eq. 17. For the $M \Phi$, we introduce their initial distribution as well as their motion velocity. Having in mind the dynamical relations and couplings between the continuum fields and individual cells in the PDE system, it is natural to introduce a chemotactic model to define the velocity field ${ }^{35,36}$ as

$$
\vec{v}=k \nabla \alpha(r, \theta, t),
$$

where $k=k(\alpha(r, \theta, t))$, a scalar function of chemoattractant. The chemoattractant $\alpha(r, \theta, t)=\gamma_{1} l_{1}(r, \theta, t)+\gamma_{2} l_{2}(r, \theta, t)+$ $\gamma_{3} e_{1}(r, \theta, t)$ contains the total tissue growth factors (TGFs) and TGFs activating enzyme type I. For this article, we only take $k=1, \gamma_{1}=\gamma_{2}=\gamma_{3}=1$ for illustrative purpose because we do not have corresponding experimental data available to compare. $\mathrm{M} \Phi$ are initially positioned on the outer wound boundary and are released at different time intervals to migrate toward the implants. $M \Phi$ cells 
influence the chemical fields as they release growth factors and enzymes, as discussed in Modeling considerations. For discrete modeling, the release is distributed around

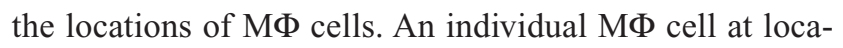
tion on the implant $\left(x_{c}, y_{c}\right)$ is modeled by a Gaussian bell weight function $w(r, \theta)=\exp \left\{\left(-\left(x-x_{c}\right)^{2}-\left(y-y_{c}\right)^{2}\right) / \sigma^{2}\right\}$. The scaling constant $\sigma$ governs the length of the radius of a sphere about that cell where the majority of the influence would lie (here we chose $\sigma=1 / \sqrt{5})$, and $\left(x_{c}, y_{c}\right)$ are the Cartesian coordinates of a cell. The total influence at a given node is determined by superposition of weight functions from all cells, ie,

$$
l=B \sum_{n=1}^{N} w_{n}(r, \theta) .
$$

Here $B$ is a constant, and $w_{n}(r, \theta)$ represents the contribution from $n$th $\mathrm{M} \Phi$ cell. We chose $B=100$ in order to maintain the same magnitude as our earlier simulation data in Su et al. ${ }^{34}$

\section{Working platform and simulator}

These differential equation models were implemented by a numerical algorithm written in Fortran code. The spatial derivatives were solved by an operator splitting method, and time derivatives were approximated by a fourth order RungeKutta method. The numerical code was then tested with several cases and compared with simulated results performed by other software. All of these tests yield nearly identical results. The calculations in this article were executed on an HP 8-core workstation platform, and results were plotted by using MATLAB. One 40-day (long-time) simulation for given initial conditions did not exceed the rate of 2.5-3 h.

\section{Results}

The computational simulations produced by this model have been useful in proving a quantitative analysis of foreign bodyassociated fibrotic reactions. Several simulation studies were carried out to determine whether the model can be used to capture the main relationship of key component variables and to predict the trend shown by these variations. Our findings are summarized as follows.

\section{The mathematical model does reflect the different timing scale and general temporal kinetics}

As in other biological experiments, foreign body fibrotic processes involve a cascade of complex dynamics and many deterministic and random factors. It would be hard to directly compare quantities of products, because large variations exist due to limitations in experimental design and detection. However, the timings of proliferation and decay of elements are relatively easy to capture and use as landmarks for reactions.

Using the parameter set we estimated in Materials and methods, and assuming the system is homogeneous in space, the governing system is composed of 11 ordinary differential equations. Based on an initial value set mimicking implantations, we solved the system numerically to obtain the temporal behavior. All variables in the system were calculated and the time courses of each variable were depicted in Figure 2. The transient behavior indicated an early spike of fibroblast and TGF- $\beta$, and collagen growth presented a slow rising curve. The timings of the kinetics were consistent with experimental observations in Table 2.

\section{Fibroblast number affects the timing of fibrotic reactions as well as collagen production}

Next, we tested the effect of initial fibroblast number change on fibrotic reactions. It is well established that fibroblast proliferation is a crucial factor and catalyst in the foreign body reactions. As shown in Figure 3, the initial fibroblast increase significantly altered the levels of collagen deposits, and the presence of fibroblasts also promoted the activation of various fibrotic factors such as TGF- $\beta$ and MФ (see more detail in $\mathrm{Su}_{\mathrm{et}} \mathrm{al}^{34}$ ). On the other hand, a modest suppression of fibroblasts is associated with the reduction of collagen production. Our results support the importance of fibroblast responses on wound-healing processes.

\section{Specific enzyme level greatly influences the extent of collagen production}

As we continued our numerical experiments to gain greater insight into the significant contributing factors in foreign body reaction process, we observed (Figure 4) that specific enzyme type I (which converts latent TGF- $\beta$ to active TGF- $\beta$ ) played an important role in kinetics. It promoted both the activation of TGF- $\beta$ and the production of fibroblasts and enzyme type II (see $\mathrm{Su}$ et $\mathrm{al}^{34}$ ). In the same study, modeling also showed that there is always an abundance of latent TGF- $\beta$. As active TGF- $\beta$ is essential to fibrotic tissue reactions, the ability to convert from latent TGF- $\beta$ to active TGF- $\beta$ may dictate the extent of fibrotic tissue reactions accompanied by the production and accumulation of collagen. 

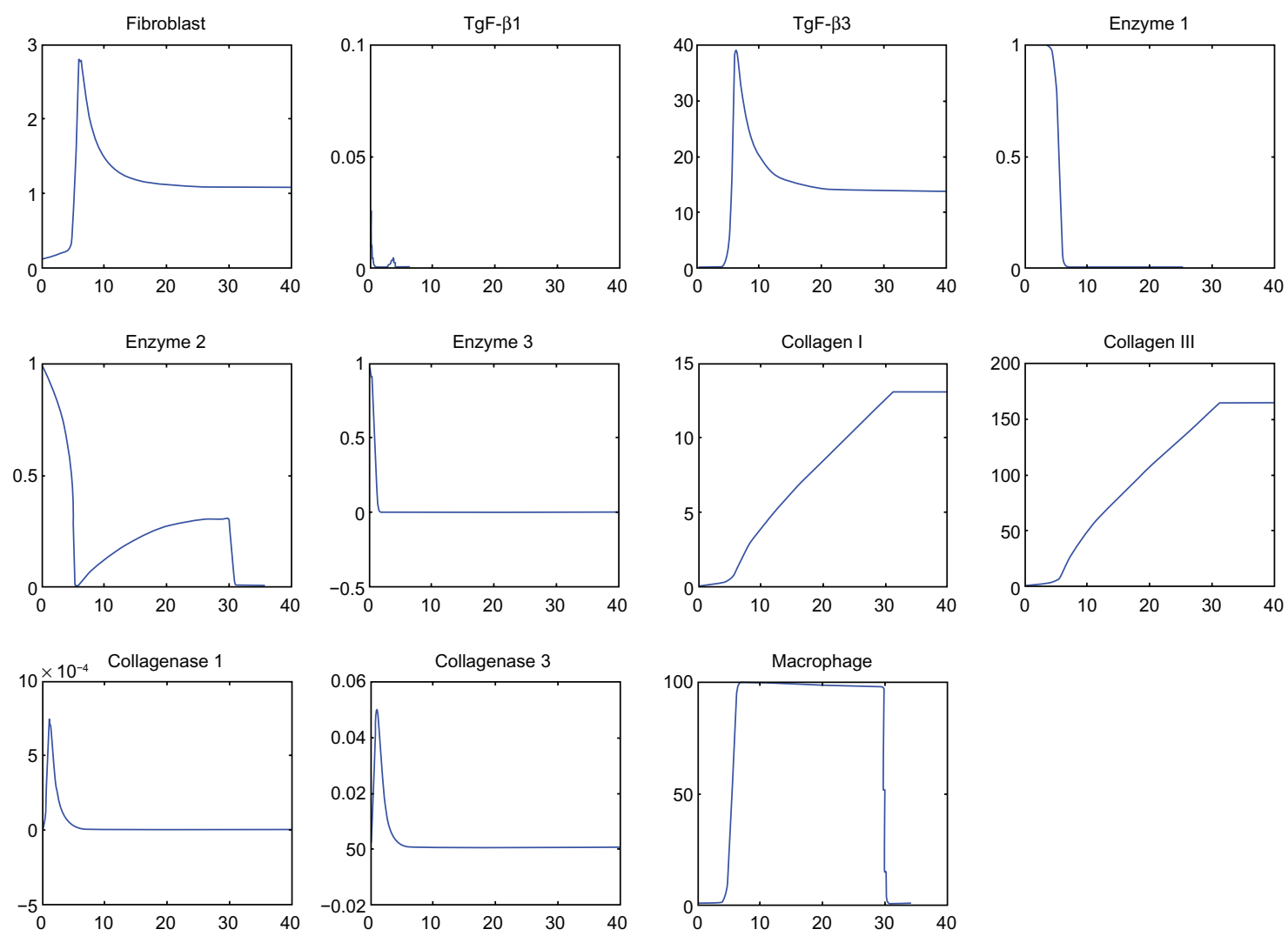

Figure 2 The simulated kinetics dynamics of variables representing collagens, collagenases, TGF- $\beta$ s, and others during the first 40 days. Abbreviation: TGF- $\beta$, transforming growth factor type $\beta$.

\section{Spatial patterns of collagen growth form a propagating wave toward the implant}

We then started to investigate simulated collagen growth near an implant in two spatial dimensions. The purpose of these simulations was two-fold. One goal was to understand how the migration of cells influences the foreign body reaction kinetics. The second was to understand if a nonhomogeneous spatial pattern alters the results of kinetics.

For our purpose, we place a circular-shaped implant (size: radius $1 \mathrm{~cm}$ ) at the center of a reaction domain (size: radius $8 \mathrm{~cm}$ ). Healthy tissue (with all variables at their normal levels) is positioned immediately adjacent to the reaction
A

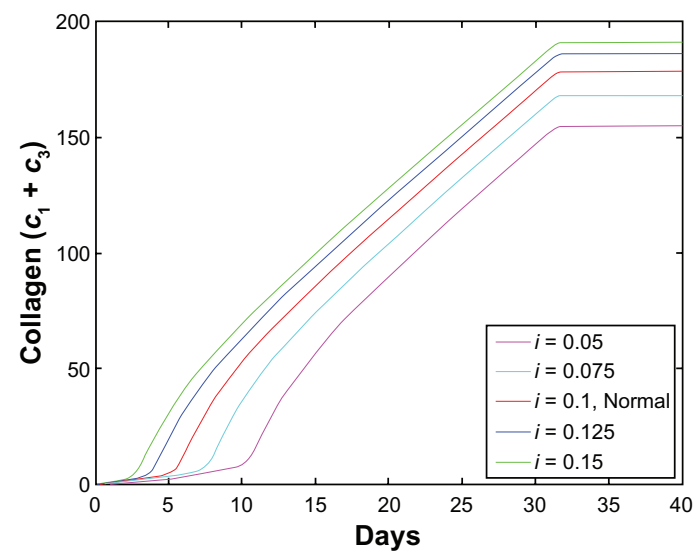

B

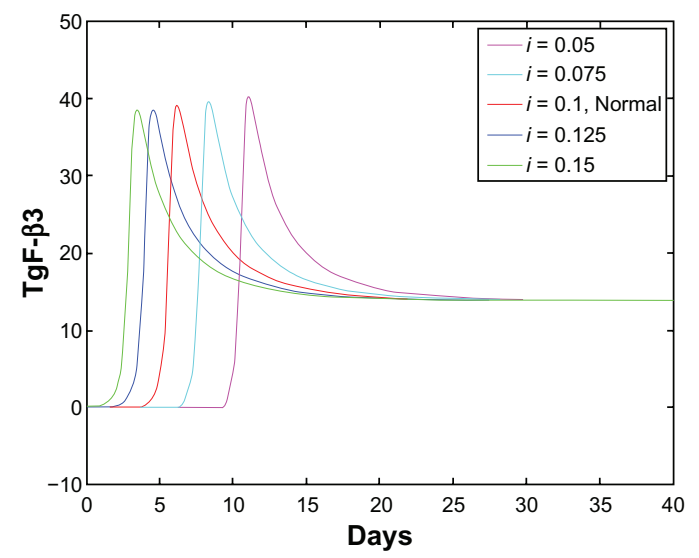

Figure 3 Influence of fibroblast numbers on $\mathbf{A}$ ) total collagen production $c_{1}+c_{3}$ and B) TGF- $\beta$ III concentration $\beta_{3}$ at different initial fibroblast levels $f$ for up to 40 days. Abbreviation: TGF- $\beta$, transforming growth factor type $\beta$. 
A

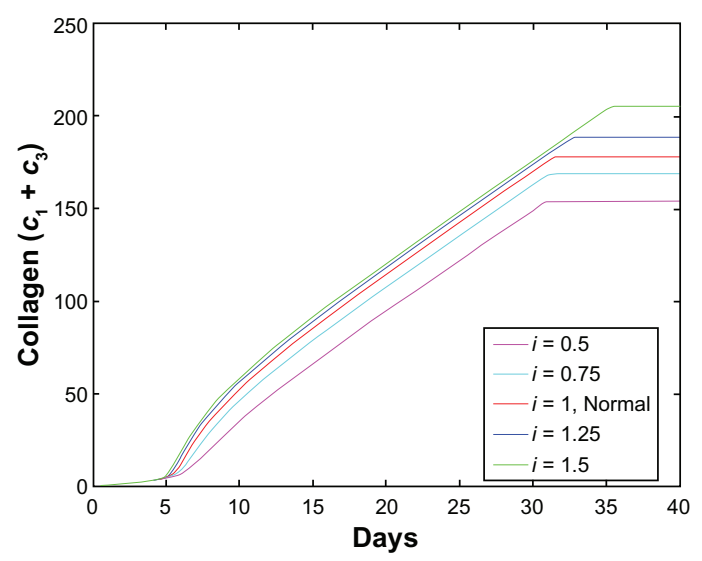

B

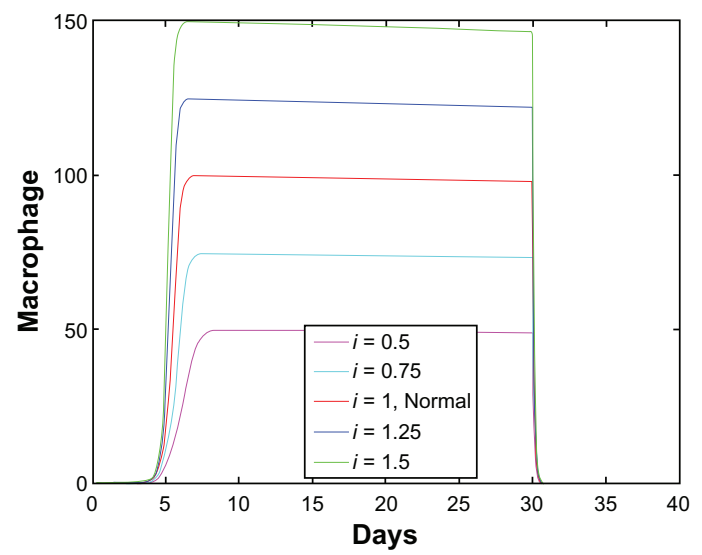

Figure 4 Effect of initial levels of enzyme type $\mathrm{I}, \mathrm{e}_{1}$, on $\mathbf{A}$ ) total collagen production $c_{1}+c_{3}$ and $\mathbf{B}$ ) MФ accumulation I for up to 40 days.

domain, and these normal cell/protein/enzyme values are imposed as boundary conditions for our system.

We first tested with symmetric initial conditions to mimic healing by migration of cells from normal tissues. After 35 days, our model showed that the collagen level

A

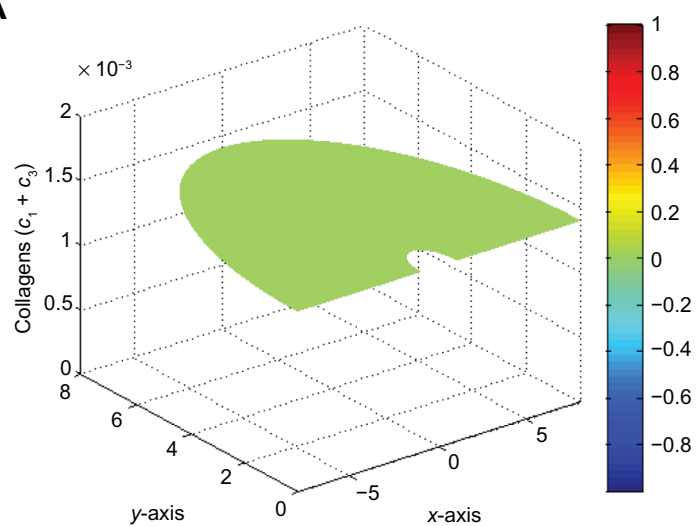

C

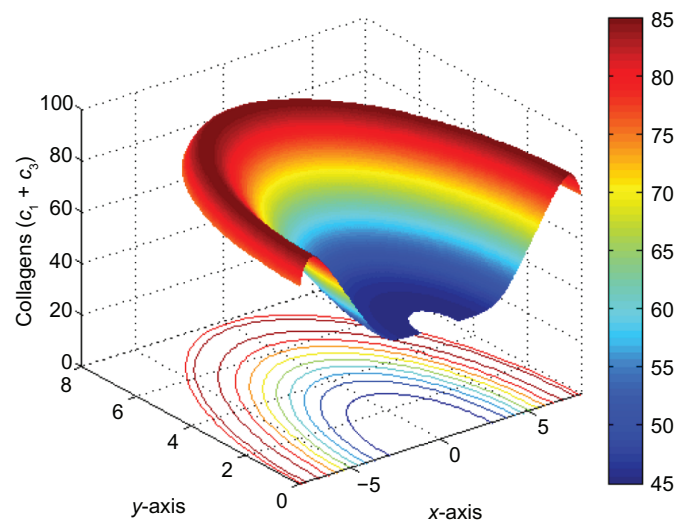

gradually flattened out around $180 \mu \mathrm{g} / \mathrm{cm}^{2}$ (Figure 5). This finding is consistent with pure temporal kinetics simulations. The implant domain was gradually filled with collagen through both activation and migration of cells. We also observed that the collagen peak did travel inward at a finite
B

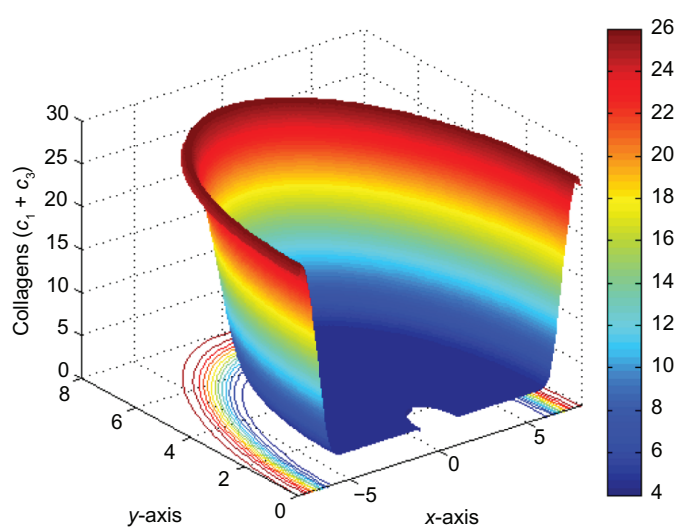

D

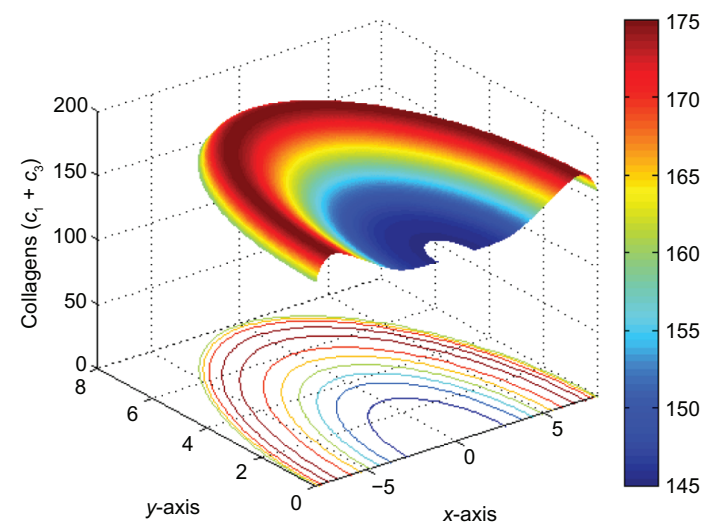

Figure 5 Three-dimensional graphs showing the levels of collagen relative to distance from the implant. A) The total collagen level, $c_{1}+c_{3}$, at $t=0$, B) at $t=5$ days, C) at $t=15$ days, and $\mathbf{D})$ at $t=35$ days. The calculation was performed in the entire domain. For better visualization, figures here and hereafter only show a half domain with half of the implant being shown as the empty half circle with radius $\mathrm{I} \mathrm{cm}$ at the location where $x$ and $y$ are zero. 
speed, but lagged far behind the propagation front of $M \Phi$, fibroblast, and others.

Subsequent tests were carried out by adding a thin layer of fibroblasts to only a portion of the implant surface. The model showed that the added layer of fibroblasts had a lasting but modest effect on the spatial pattern of collagen (as reported in Supplementary form S1) as well as other ingredients of reactions. We further observed that the migration has a limited impact on spreading the spatial difference of collagen production. The overall kinetic changes were mainly due to fibroblast activation.

\section{Continuous-discrete cell model reflects spatial variation and clustering of cells}

The results of our multiscale continuous-discrete model simulation further revealed that $\mathrm{M} \Phi$ cells tend to cluster together near the implant surface. This is consistent with the formation of foreign body giant cells adjacent to the implants, as observed in experiments (summarized in Figure 6A, B and their text). This phenomenon is supported by our model.

Even though the total trend of growth dynamics was already reflected in the continuum model, the multiscale model showed that cells tend to pack together around the implant and form several clusters. In particular, a large density of collagen was found near the implants (Figure 6A, B). The uneven distribution pattern of collagen layers can

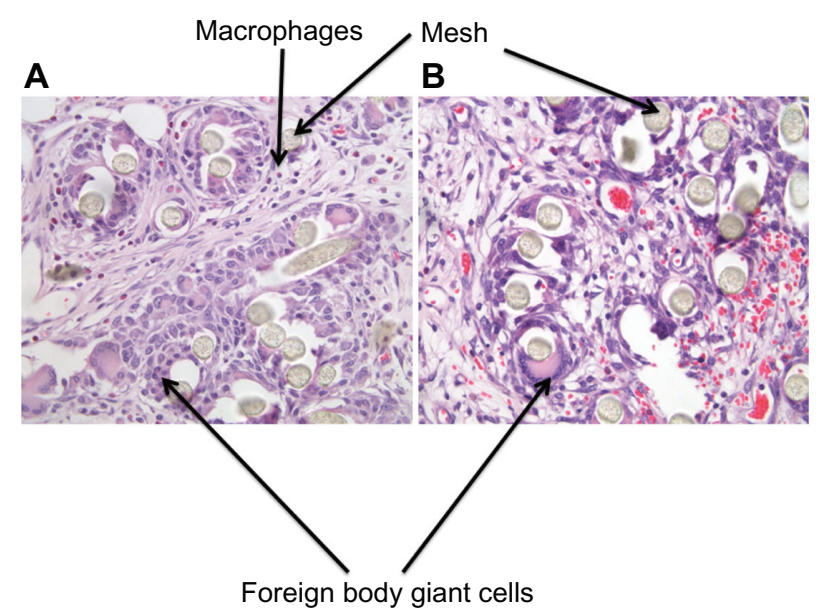

Figure 6 A) A photo of implanted tissue (left panel). Large numbers of $M \Phi$ reside adjacent to polymer mesh (dark circular object), and they gradually form a cluster of inflammatory cells and/or foreign body giant cells. Foreign body giant cells are cells with multinuclei and are a feature of foreign body reactions. Fibroblasts are elongated cells and are located between inflammatory cells and normal tissues. They are present in the middle of the photo. B) A locally enlarged picture of inflammatory tissue near the implant (right panel). Polymer mesh implants are surrounded by $M \Phi$ and foreign body giant cells. The activation of the $М \Phi$ can also lead to new blood vessel formation and angiogenesis, shown as red dots in the photo (angiogenesis feature is not included in this study). As part of the chronic foreign body reactions, fibroblasts produce collagen and form fibrotic capsules surrounding medical implants or foreign objects. be more visible when the number of cells is small in our multiscale model. Our simulations clearly have given an account of the time evolution process, which could not be modeled by the continuum model.

In simulations, we placed 10 groups of $12 \mathrm{M} \Phi$ in different positions on the outer wound boundary, and they were moved one group after another. Different groups of the $М \Phi$ were released every 3 days, ie, $t=3$ days. МФ cells were evenly distributed in the outer boundary (normal tissue). The time evolution of specific enzyme type II released by the presence of $M \Phi$ is revealed in Figure 7. After 1 day, many $М \Phi$ arrived at the implant surface and stayed there for the entire period (Figure 7A). As expected, $М \Phi$ propagated at a much faster speed than the collagen. At day 30, about $120 \mathrm{M} \Phi$ arrived at the implant's surface, and they formed 4-5 clusters, each containing 20-30 MФ cells. Meanwhile, the total collagen levels at the same time interval were depicted (Figure 8). We found that the collagen took a much longer time to proliferate and to migrate to the implant. It was not until around day 5-7 that the collagen started to grow and the slow rising curve accumulated to a higher level as more MФ arrived. They did, however, show a similar cluster pattern in collage level.

Because the number of $\mathrm{M} \Phi$ is considered large (120 cells) in this simulation, when the total collagen volume in the entire domain is compared with both the continuum and multiscale models, the multiscale simulations show some agreement with our continuum model. However, the distribution of collagen was not as uniform in the discrete model. We see in Figure 8D that the collagen level near the implant was near $1800 \mu \mathrm{g} / \mathrm{cm}^{2}$ (in comparison with an average of 200 in the continuum model), but over the majority of the domain, the collagen level was close to 200 . The multiscale model compares more favorably with experimental observation, where more compact narrow regions of collagen films were observed.

We also simulated the migration of individual $M \Phi$ appearing one cell at a time. The results are reported in Supplementary form S2. With a small number of $M \Phi$, the role of individual M $\Phi$ is more prominent. Each $М \Phi$ contributed to a virtually equivalent peak of collagen mass. At the end of 30 days, the collagen peaks merged together and accumulated to a higher peak. The discrete model supports the individualized role of each $\mathrm{M} \Phi$ and their contributions to collagen production.

\section{Discussion}

Our model can be used as a powerful tool for a variety of investigations. By varying different parameters, the 


\section{A}

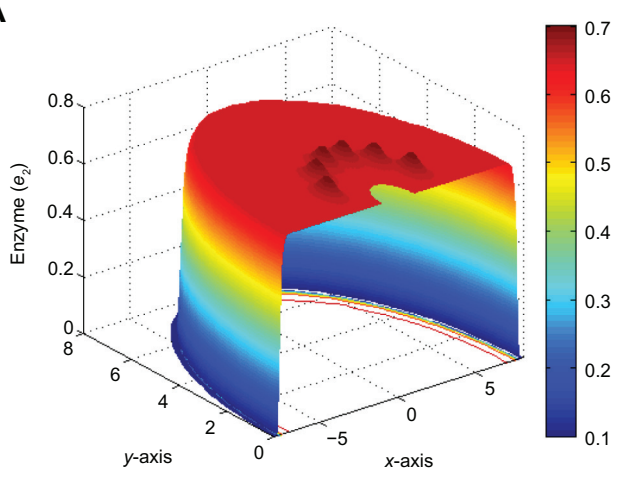

C

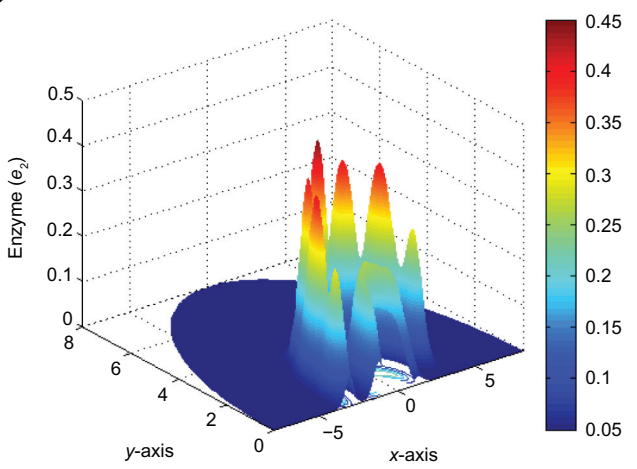

B

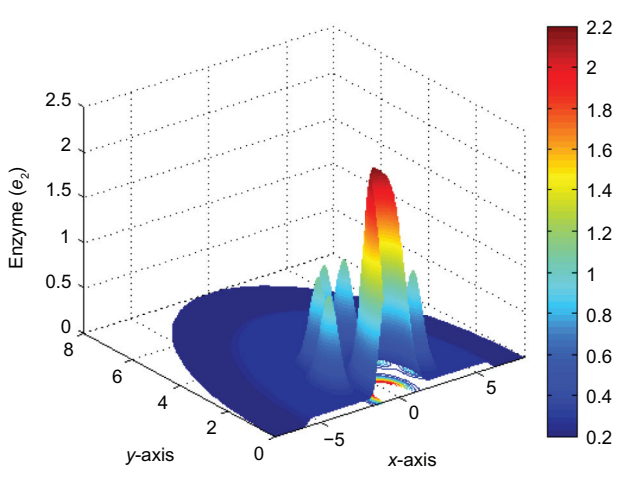

D

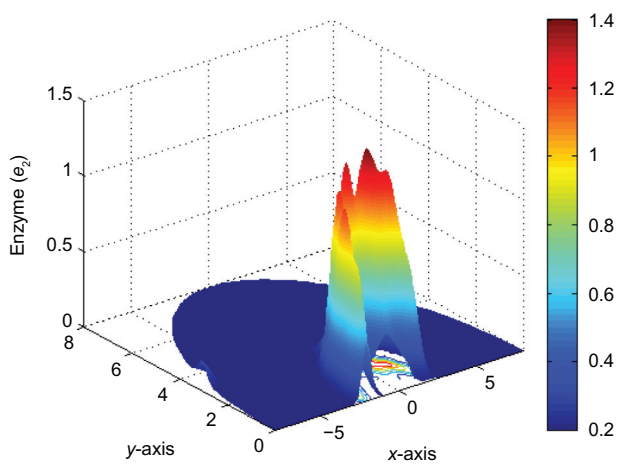

Figure 7 Snapshots of the evolution of specific enzyme type II $\left(\mathrm{e}_{2}\right)$ for $\left.\left.\left.\mathbf{A}\right) t=\mathrm{I}, \mathbf{B}\right) t=5, \mathbf{C}\right) t=15$, and $\left.\mathbf{D}\right) t=30$ days. The propagation was initiated from a periodical release of $12 M \Phi$ in each group over 3 days. The release locations were evenly distributed. But positions were alternated each time to be the midpoint of last distribution on the outer boundary of the wound (from healthy tissue).

A

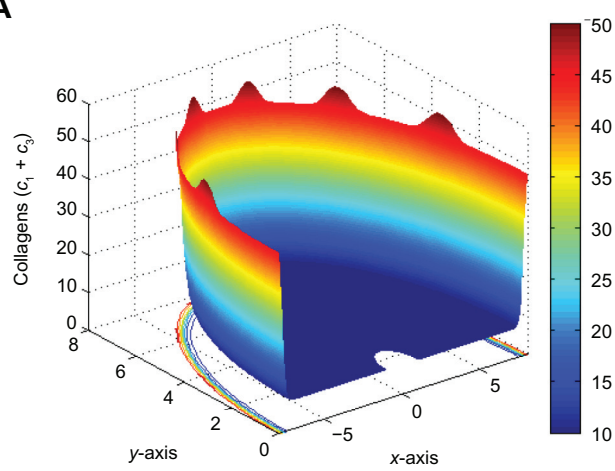

C

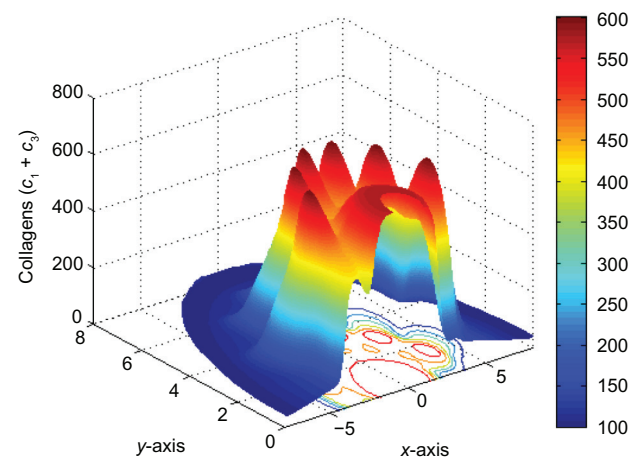

B

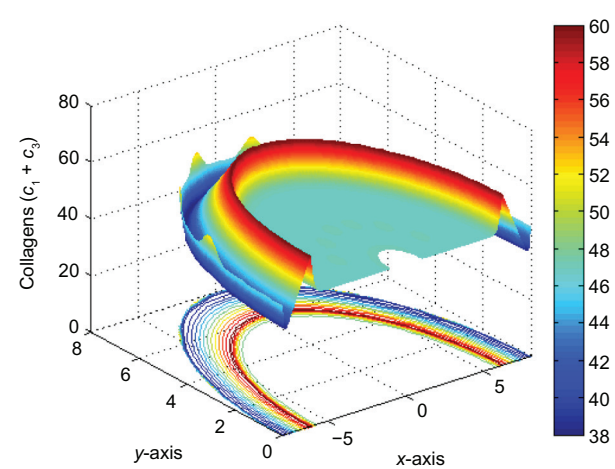

D

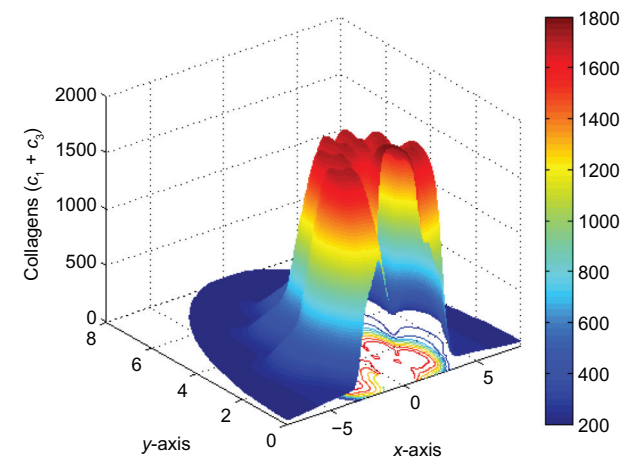

Figure 8 Snapshots of evolution of total collagens, $c_{1}+c_{3}$, for $\left.\left.\left.\mathbf{A}\right) t=1, \mathbf{B}\right) t=5, \mathbf{C}\right) t=15$, and $\left.\mathbf{D}\right) t=30$ days. The collagen distributions were concentrated on several clusters of foreign body giant cells, and they accumulated near the surface of implant and formed a thin film at 30 days. This scenario was very different from our earlier continuum model prediction in Figure 5, where cells were treated as a continuum. 
simulations do allow us to search pathways for collagen growth and the significant factors affecting such reactions.

\section{Collagens are not sensitive to most chemical levels}

We systematically tested the effects of initial chemical level changes on collagen production and found most of them were not correlated to such responses. For example, we experimented with changes of initial latent TGF- $\beta$ in the temporal model. Other than for an initial transient period, the latent level did not change the kinetics very much. Because active TGF- $\beta$ s are known to be significant contributors, ${ }^{7}$ the conversion rate of active TGF- $\beta$ s (but not latent TGF- $\beta$ s) was important for overall kinetics. This was also shown by the significant contribution of specific enzyme type I in our earlier work. ${ }^{34}$ It is also possible that collagen production would be reduced by decreasing the release of specific enzyme type I (hence reducing the conversion rate of latent to active TGF- $\beta$ s). However, further study is needed to explore such a strategy.

\section{Chemicals added at early stages of reactions can be useful to change kinetics}

Our simulation studies have shown that adding latent TGF- $\beta$ s at different days of the fibrotic reactions affects the overall collagen production. These results can be helpful in designing treatments for suppressing inflammation and can be readily implemented in experiments. We observed in Figure 9A, B that addition of latent growth factor TGF- $\beta$ I helped to speed up the proliferation of $\mathrm{M} \Phi$ and increase collagen growth.
However, addition of TGF- $\beta$ I after the fifth day showed little or no effect (the 10th-day curve completely overlaps with the normal curve). This is consistent with the fact that growth TGF- $\beta$ s mainly play a role in early inflammation, but the collagen growth process in later stages does not have much dependency on TGF- $\beta$ s. ${ }^{1}$

\section{Collagen growth mainly depends on fibroblast activities}

Finally, we point out that fibroblasts and their various reaction rates are sensitive contributors to the production of collagen. As shown in Figure 10 (for $a_{1}$ ), changes in $a_{1}-a_{4}$ resulted in significant variations in collagen production. Two collagen reaction rates $B_{22}, B_{24}$ (shown in Figure 10 for $B_{24}$ ) are also related to fibroblast reactions with enzymes. This is consistent with the fact that major sources of procollagens are from fibroblast secretions. ${ }^{9}$

\section{Conclusion}

Despite increasing popularity in contemporary medicine, medical implants often trigger different extents of foreign body reactions. Substantial research efforts have been placed to uncover the processes governing such reactions. Unfortunately, most of the in vitro and in vivo biological assays can only be used to study one or two variables. Therefore, the complex interactions between different cells and cellular products (growth factors and cytokines) cannot be determined using traditional experimental approaches. The recent advancement of computational biology has shown that it is possible that mathematical models can be built to

B

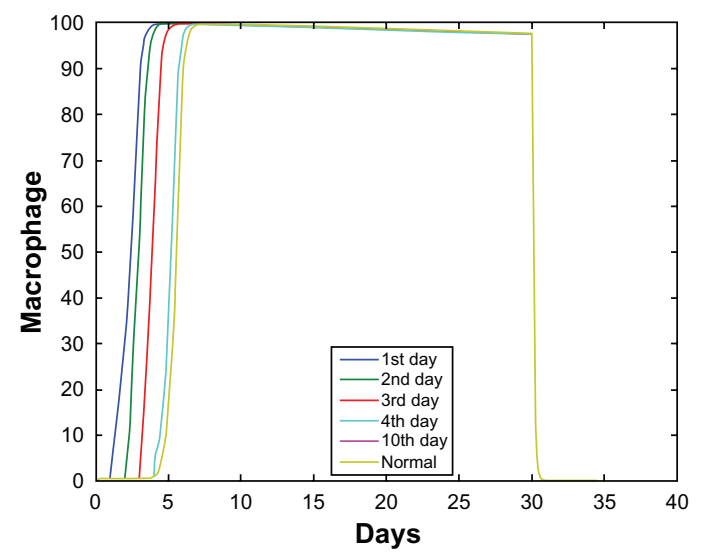

B

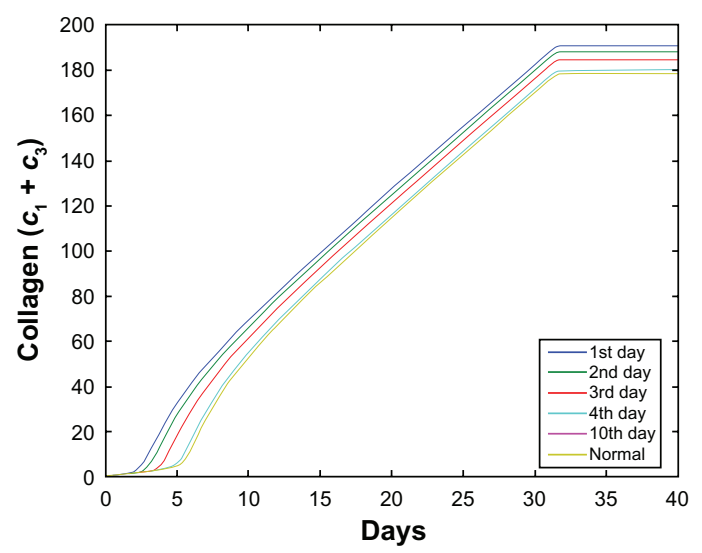

Figure 9 Effect of latent growth factor TGF- $\beta$ I, $I_{1}$. A) The MФ level for up to 40 days, as latent growth factor TGF- $\beta$ I, $I_{1}$ was added to reaction at different days. B) The total collagen level for up to 40 days for the same treatments. Different day represents the time for adding latent TGF- $\beta$ (quantity is same as healthy tissue level), and "normal" stands for no addition of TGF- $\beta$ I during process.

Abbreviation: TGF- $\beta$, transforming growth factor type $\beta$. 
A

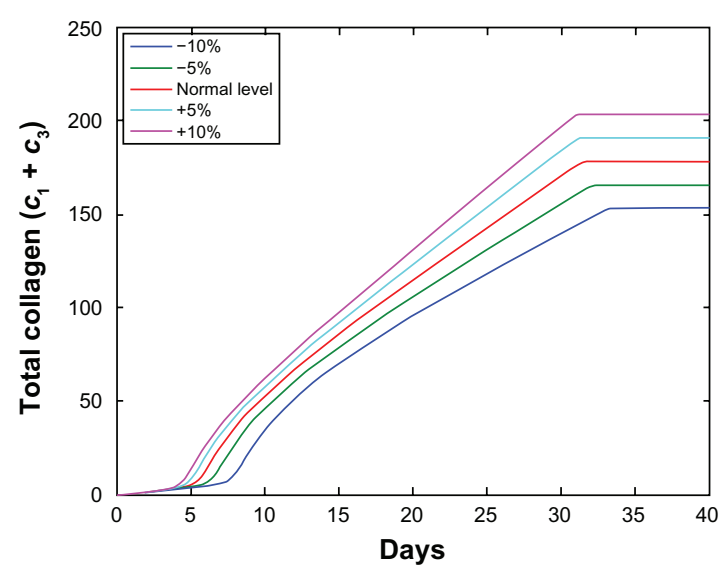

B

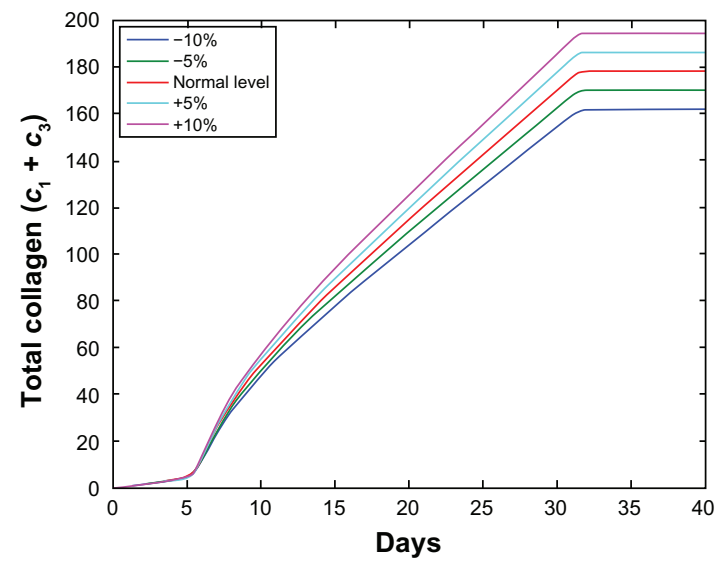

Figure 10 A) The total collagen level for up to 40 days at different levels of fibroblast reaction rate $a_{1}$. B) The sensitivity of total collagen level toward collagen reaction rate $B_{24}$. Different \% level represents the variation of the parameter in either direction.

simulate complex reactions and interactions. To quantitatively study the processes governing biomaterial-mediated fibrotic reactions, we have built a mathematical model with capability to predict the trends of macrophage migration, collagen production, and enzyme regulation in foreign body reaction processes. Our model is built based principally on biochemical mechanisms and calibrated with experimental data of collagen production. Some system parameters were based on known biochemical data in literature, and others were determined by fitting simulated results and experimental data via a series of numerical simulations.

The implementation of the mathematical model has many advantages. First, our results support that this model can be used to investigate multiple variables and complex interactions in a systematic way. Second, this model can be modified to simulate different types of inflammatory and fibrotic diseases. Third, by comparing experimental observation and simulation outcomes, we would be able to determine the potential interactions between many variables, including cells and cellular products, on different stages of foreign body reactions. Fourth, mathematical modeling allows us to expand the experimental single variable and single time point observations to multiscale and kinetic responses. This capability allows us to predict the potential outcomes by changing any one or multiple variables. Finally, by further developing this mathematical model, we may be able to use it to identify the critical variable and time point in which treatments can be made to alter fibrotic tissue reactions with favorable outcomes. For example, it is well established that excessive fibrotic reactions would impede drug release from some medical devices, whereas insufficient fibrotic reactions are responsible for the prolonged recovery of ligament transplantation. It is our hope that the mathematical model can be further developed and calibrated to simulate complex tissue reactions to medical implants. Such models will improve our understanding and perhaps uncover unknown factors governing foreign body reactions. Furthermore, this model may also help to explore various treatments for foreign body responses. By incorporating experimental results obtained from material with different physical and chemical properties, this model may be used to engineer biomaterials for triggering desired tissue reactions.

\section{Acknowledgments}

This work is supported by National Institutes of Health grant \# 1R01EB007271.

\section{Disclosure}

The authors report no conflicts of interest in this work.

\section{References}

1. Tang L, Eaton JW. Inflammatory responses to biomaterials. Am J Clin Pathol. 1995;103(4):466-471.

2. Tang L, Eaton JW. Natural responses to unnatural materials: a molecular mechanism for foreign body reactions. Mol Med. 1999;5(6):351-358.

3. Tang L, Hu W. Molecular determinants of biocompatibility. Expert Rev Med Devices. 2005;2(4):493-500.

4. McDonald JA. Fibronectin: a primitive matrix. In: Clark RAF, Henson PM, editors. The Molecular and Cellular Biology of Wound Repair. New York: Plenum Press; 1988:405-436.

5. Appling WD, O'Brien WR, Johnston DA, Duvic M. Synergistic enhancement of type I and III collagen production in cultured fibroblasts by transforming growth factor- $\beta$ and ascorbate. FEBS Lett. 1989;250(2):541-544.

6. Goldberg B. Kinetics of processing of type I and type III procollagens in fibroblast cultures. Proc Natl Acad Sci U S A. 1977;74(8):3322-3325.

7. Martin P, Hopkinson-Woolley J, McCluskey J. Growth factors and cutaneous wound repair. Prog Growth Factor Res. 1992;4(1): 25-44. 
8. Streuli CH, Schmidhauser C, Kobrin M, Bissell MJ, Derynck R. Extracellular matrix regulates expression of the TGF- $\beta 1$ gene. $J$ Cell Biol. 1993;120(1):253-260.

9. Roberts AB, Sporn MB. The transforming growth factor- $\beta$ s. In: Sporn MB, Roberts AB, editors. Peptide Growth Factors and Their Receptors. Berlin: Springer-Verlag; 1990:419-472.

10. Miller GJ, Gay S. Collagen structure and function. In: Cohen IK, Diegelmann RF, Lindablad WJ, editors. Wound Healing: Biochemical and Clinical Aspects. Philadelphia (PA): W.B. Saunders Co; 1992:63-76.

11. Stricklin GP, Eisen AZ, Bauer EA, Jeffrey JJ. Human skin fibroblast collagenase: chemical properties of precursor and active forms. Biochemistry. 1978;17(12):2331-2337.

12. Anderson JM. Mechanisms of inflammation and infection with implanted devices. Chapter 4. Cadiovasc Pathol. 1993;2(3 Suppl 1): 33-41.

13. Mosser DM, Edwards JP. Exploring the full spectrum of macrophage activation. Nat Rev Immunol. 2008;8(12):958-969.

14. Fadok VA, Bratton DL, Konowal A, Freed PW, Westcott JY, Henson PM. Macrophages that have ingested apoptotic cells in vitro inhibit proinflammatory cytokine production through autocrine/ paracrine mechanisms involving TGF- $\beta$, PGE2, and PAF. $J$ Clin Invest. 1998;101(4):890-898.

15. Clancy RM, Buyon JP. Clearance of apoptotic cells: TGF- $\beta$ in the balance between inflammation and fibrosis. J Leukoc Biol. 2003;74(6):959-960.

16. Loke P, Gallagher I, Nair MG, et al. Alternative activation is an innate response to injury that requires $\mathrm{CD} 4+\mathrm{T}$ cells to be sustained during chronic infection. J Immunol. 2007;179(6):3926-3936.

17. Gordon S, Taylor PR. Monocyte and macrophage heterogeneity. Nat Rev Immunol. 2005;5(12):953-964.

18. Schugart RC, Friedman A, Zhao R, Sen CK. Wound angiogenesis as a function of tissue oxygen tension: a mathematical model. Proc Natl Acad Sci U SA. 2008;105(7):2628-2633.

19. Tang L, Wu Y, Timmons RB. Fibrinogen adsorption and host tissue responses to plasma functionalized surfaces. $J$ Biomed Mater Res. 1998;42(1):156-163.

20. Dee KC, Puleo DA, Bizios R. Wound healing. In: Dee KC, Puleo DA, Bizios R, editors. An Introduction to Tissue-Biomaterial Interactions. Hoboken (NJ): John Wiley and Sons Inc; 2002:165-214.

21. Anderson JM. Inflammation and the foreign body response. Probl Gen Surg. 1994;11:147-160.
22. Mantzaris NV, Webb S, Othmer HG. Mathematical modeling of tumorinduced angiogenesis. J Math Biol. 2004;49(2):111-187.

23. Dale PD, Sherratt JA, Maini PK. A mathematical model for collagen fibre formation during foetal and adult dermal wound healing. Proc Biol Sci. 1996;263(1370):653-660.

24. Lemon G, Howard D, Tomlinson MJ, et al. Mathematical modelling of tissue-engineered angiogenesis. Math Biosci. 2009;221(2):101-120.

25. Dallon JC, Sherratt JA, Maini PK. Modeling the effects of transforming growth factor- $\beta$ on extracellular matrix alignment in dermal wound repair. Wound Repair Regen. 2001;9(4):278-286.

26. Dale PD, Sherratt JA, Maini PK. Role of fibroblast migration in collagen fiber formation during fetal and adult dermal wound healing. Bull Math Biol. 1997;59(6):1077-1100.

27. Krummel TM, Michna BA, Thomas BL, et al. Transforming growth factor beta (TGF-beta) induces fibrosis in a fetal wound model. J Pediatr Surg. 1988;23(7):647-652.

28. Wakefield LM, Smith DM, Flanders KC, Sporn MB. Latent transforming growth factor- $\beta$ from human platelets. A high molecular weight complex containing precursor sequences. J Biol Chem. 1988;263(16):7646-7654.

29. Sinclair RD, Ryan TJ. Proteolytic enzymes in wound healing: the role of enzymatic debridement. Australas J Dermatol. 1994;35(1):35-41.

30. Owen MR, Sherratt JA. Mathematical modelling of macrophage dynamics in tumours. Math Models Methods Appl Sci. 1999;9(4):513-540.

31. Tang L, Jennings TA, Eaton JW. Mast cells mediate acute inflammatory responses to implanted biomaterials. Proc Natl Acad Sci U S A. 1998;95(15):8841-8846.

32. Jeffrey JJ. Collagen synthesis and degradation in the uterine deciduoma: regulation of collagenase activity by progesterone. Coll Relat Res. 1981;1(3):257-268.

33. Su J, Gonzales HP, Tang L. Modeling and simulation of foreign body reactions to neural implants. In: Wang R, Gu F, Shen E, editors. Advances in Cognitive Neurodynamics, Proceedings of the International Conference on Cognitive Neurodynamics. New York: Springer; 2008:879-883.

34. Su J, Gonzales HP, Todorov M, Kojouharov H, Tang L. A mathematical model for foreign body reactions in 2D. Int J Comput Math. In press 2009.

35. Murray JD. Mathematical Biology II: Spatial Models and Biomedical Applications. 3rd ed. New York, Berlin: Springer-Verlag; 2002.

36. Keller EF, Segel LA. Model for chemotaxis. J Theor Biol. 1971; 30(2):225-234. 


\section{Appendix}

\section{Governing equations}

We consider the foreign body reactions in a two-dimensional space. The physical setting is as follows:

- $G_{0}=\{(r, \theta), 0 \leq r \leq 1,0 \leq \theta<2 \pi\}$ is the location of implant,

- $G_{1}=\{(r, \theta), 8 \leq r<\infty, 0 \leq \theta<2 \pi\}$ is the normal surrounding tissue,

- $G=\{(r, \theta), 1 \leq r \leq 8,0 \leq \theta<2 \pi\}$ is the physical implant domain where foreign body reactions take place. The domain $G$ serves as our computational domain.

We now describe the model in two spatial dimensions under the assumption that Eqs. 4, 5, 9, 10, 13, and 14 reach their equilibria instantaneously. The governing equations with diffusion are:

$$
\begin{gathered}
\frac{\partial f}{\partial t}=D_{1} \nabla^{2} f+\left(A_{1}+B_{2} e_{1} l_{1}+B_{3} e_{1} l_{3}\right) \\
f\left(1-\frac{f}{k_{1}}\right)-A_{4} f, \\
\frac{\partial l_{1}}{\partial t}=D_{2} \nabla^{2} l_{1}+\frac{A_{5} f l_{1}}{1+A_{6} l_{3}+A_{7} l_{1}}-A_{8} l_{1}-A_{16} e_{1} l_{1}, \\
\frac{\partial l_{3}}{\partial t}=D_{3} \nabla^{2} l_{3}+\frac{A_{9} f l_{3}}{1+A_{10} l_{3}}-A_{11} l_{3}-A_{17} e_{1} l_{3}, \\
\frac{\partial e_{1}}{\partial t}=-e_{1}\left(A_{16} l_{1}+A_{17} l_{3}\right), \\
\frac{\partial e_{2}}{\partial t}=-e_{2} f\left[\frac{B_{14}+e_{1}\left(B_{16} l_{1}+B_{17} l_{3}\right)}{A_{23}+A_{18} e_{3}}\right. \\
\left.+\frac{B_{15}+e_{1}\left(B_{19} l_{1}+B_{20} l_{3}\right)}{A_{27}+A_{19} e_{3}}\right]+B_{36} l,
\end{gathered}
$$

$$
\begin{aligned}
\frac{\partial e_{3}}{\partial t}=-e_{3} f & {\left[\frac{B_{34} c_{1}}{\left(1+e_{1}\left(A_{23} l_{1}+A_{18} l_{3}\right)\right)\left(A_{35}+A_{40} e_{3}\right)}\right.} \\
& \left.+\frac{B_{35} c_{3}}{\left(1+e_{1}\left(B_{19} l_{1}+B_{20} l_{3}\right)\right)\left(A_{39}+A_{41} e_{3}\right)}\right], \\
\frac{\partial c_{1}}{\partial t}= & \frac{B_{22}+A_{28} e_{1}\left(l_{1}+C_{1} l_{3}\right)}{A_{27}+A_{19} e_{2}} f e_{2}-A_{29} s_{1} c_{1}, \\
\frac{\partial c_{3}}{\partial t}= & \frac{B_{24}+A_{30} e_{1}\left(l_{1}+C_{4} l_{3}\right)}{A_{27}+A_{19} e_{2}} f e_{2}-A_{31} s_{3} c_{3}, \\
\frac{\partial s_{1}}{\partial t}= & \frac{B_{26}}{\left(1+e_{1}\left(B_{27} l_{1}+B_{28} l_{3}\right)\right)\left(A_{35}+A_{40} e_{3}\right)} \\
& \times f c_{1} e_{3}-A_{43} s_{1}+B_{37} \cdot l, \\
\frac{\partial s_{3}}{\partial t}= & \frac{B_{30}}{\left(1+e_{1}\left(B_{31} l_{1}+B_{32} l_{3}\right)\right)\left(A_{39}+A_{41} e_{3}\right)} \\
& \times f c_{3} e_{3}-A_{45} s_{3}+B_{38} \cdot l, \\
\frac{d l}{d t}= & D_{6} \nabla^{2} l+B_{39}\left(e_{1} l_{1}+e_{1} l_{3}\right) l \frac{\left(N_{n}+N_{e}\right)}{N_{n}+l} \\
& -B_{40} l-B_{41} \sigma_{0}(t-30) l .
\end{aligned}
$$

where we assume all the functions are defined and smooth in a central circular annulus $G=\{(r, \theta), 1 \leq r \leq 8,0 \leq \theta<$ $2 \pi\}, \sigma_{0}$ stands for Heaviside function, and $\nabla^{2}=\partial^{2} / \partial^{2} r+(1 / r)$ $(\partial / \partial r)+\left(1 / r^{2}\right)\left(\partial^{2} / \partial \theta^{2}\right)$ is Laplacian in polar coordinates. 


\section{Supplementary form SI}

As discussed in the main text (Spatial patterns of collagen growth form a propagating wave toward the implant), some ingredients, such as fibroblast level, can create a long-lasting impact and unevenness in the collagen deposit. We first experimented with implants that were partially coated with various active ingredients of foreign body reactions. The overall purpose of these experiments was to study the effect of spatially heterogeneous patterns of implant coating on fibroblast responses. We experimented by adding a thin layer of fibroblasts to the left half of the circle, on the implant surface,

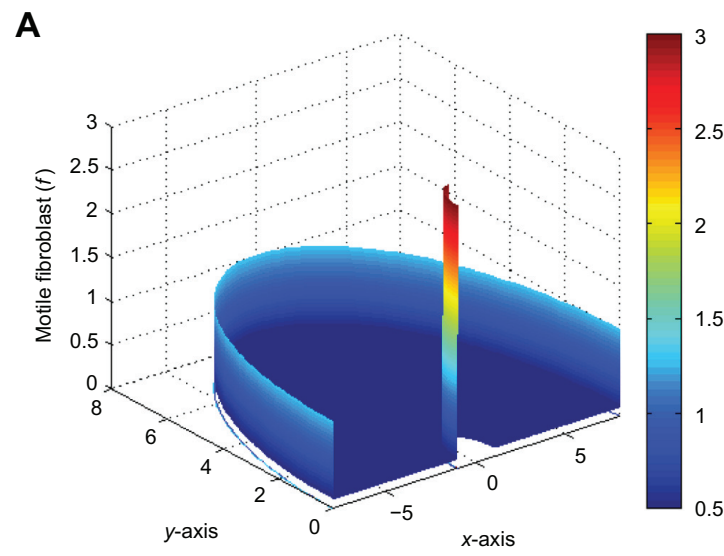

C

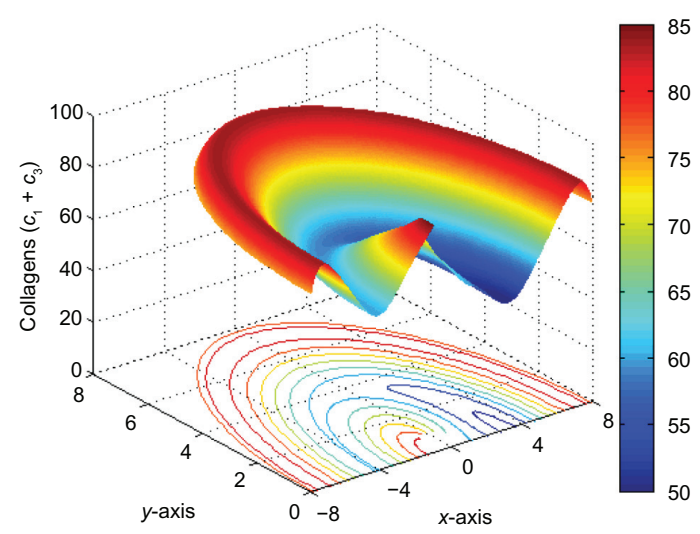

and leaving the right half of the circle unchanged. Our results show that the added fibroblast layer did have lasting impacts in changing the spatial pattern of collagen (demonstrated in Figure S1) as well as other ingredients of fibrotic reactions. On the other hand, the presence of additional fibroblasts only slightly altered the final collagen level (as seen in the mild peak localized at the implant site, Figure S1). Further studies show that the migration has minimal impact in spreading the spatial difference of collagen production, demonstrating that fibroblast activation is mainly responsible for the overall kinetic changes of collagen production.

B

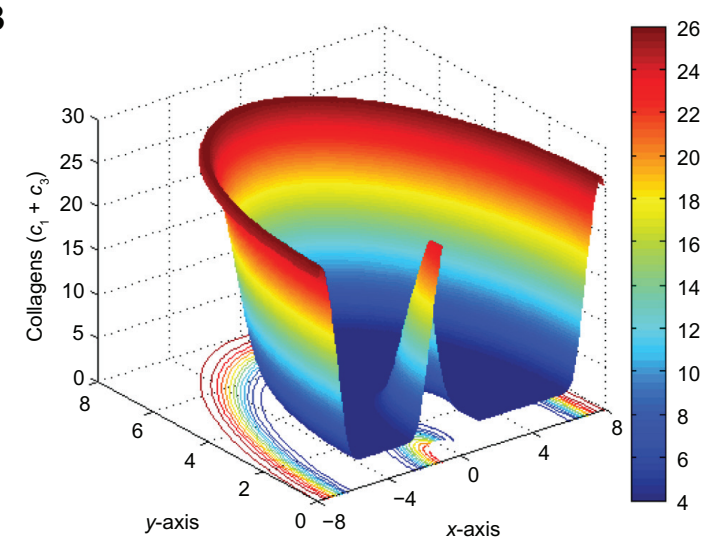

D

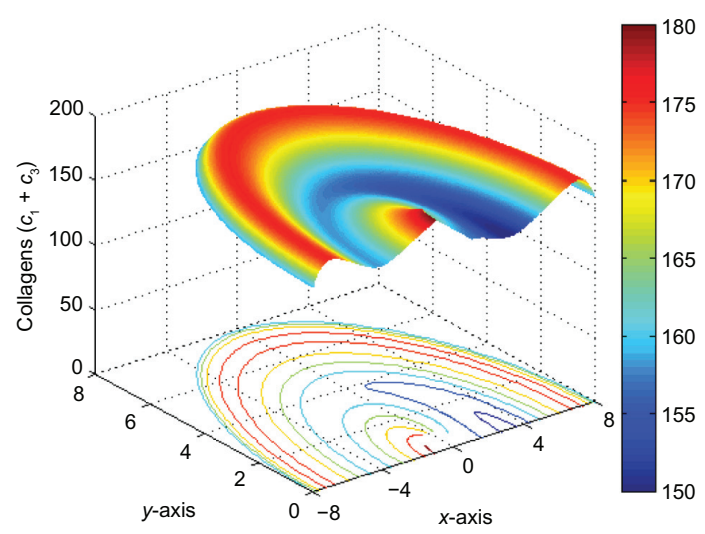

Figure SI A) The initial distribution of fibroblast $f($ at $t=0)$. The total collagen level, $c_{1}+c_{3}$, is shown $\left.\mathbf{B}\right)$ at $t=5$ days, $\left.\mathbf{C}\right)$ at $t=15$ days, and $\left.\mathbf{D}\right)$ at $t=35$ days. 


\section{Supplementary form S2}

Using our simulation model, we studied the influence of M $\Phi$ on the extent of collagen production. Specifically, we controlled the appearance of $\mathrm{M} \Phi$ at 1 cell per 0.5 day. Interestingly, the low number of $\mathrm{M} \Phi$ substantially increases the importance of each cell in the overall response. As shown in Figure S2, each M $\Phi$ produced small amounts of specific enzyme type II, reflected by small peaks surrounding the center implants (Figure S2 A-C). With increased time, M $\Phi$ migrated toward the implants and produced a large amount of the specific enzyme type II near the implant (Figure S2D). By monitoring the collagen level with time,

A

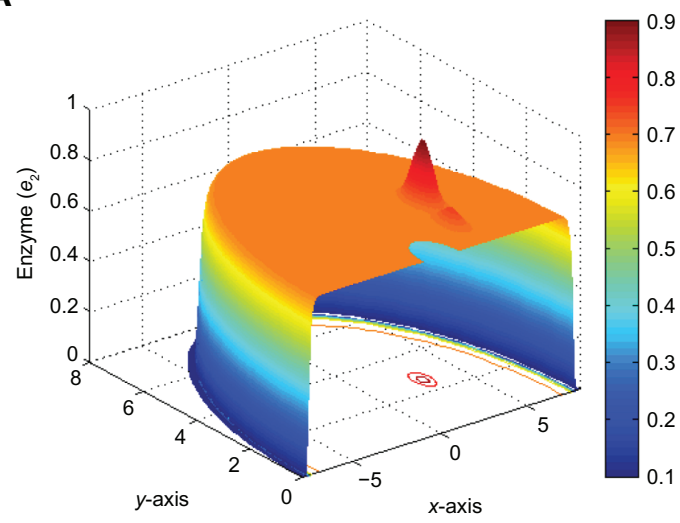

C

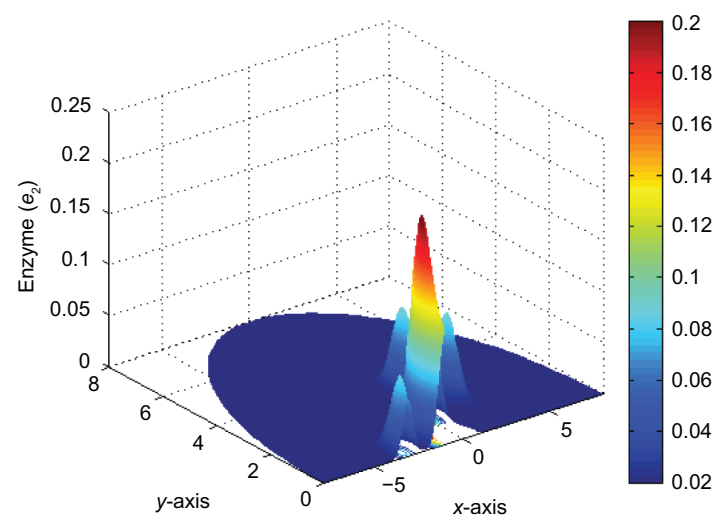

we have also observed a similar phenomenon that collagen production initially occurred on the outer ring of the fibrotic tissue (Figure S3 A). With the migration of $M \Phi$ toward medical implants, the area with maximal collagen production also moved toward the implants (Figure S3 $\mathrm{B}, \mathrm{C})$. At the end of 30 days, the area of maximal collagen production is found adjacent to the implants (Figure S3 D). However, it should be noted that the merge of specific enzyme type II peaks occurs just before that of collagen production. Our results support that this discrete model can be used to depict the role and influence of individual $\mathrm{M} \Phi$ on collagen production.

B

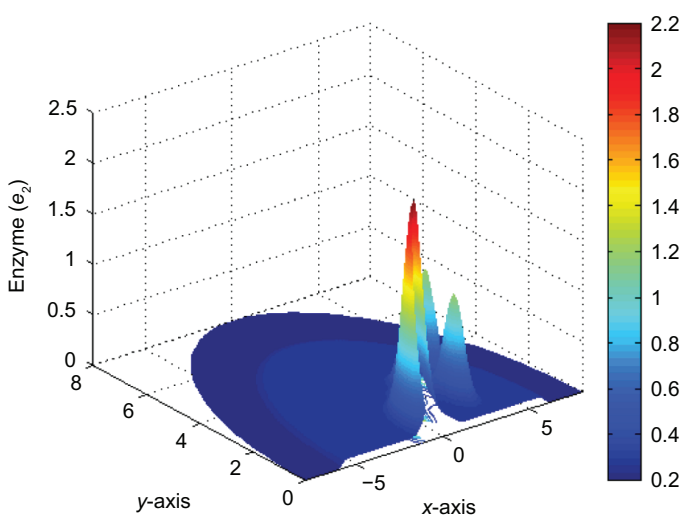

D

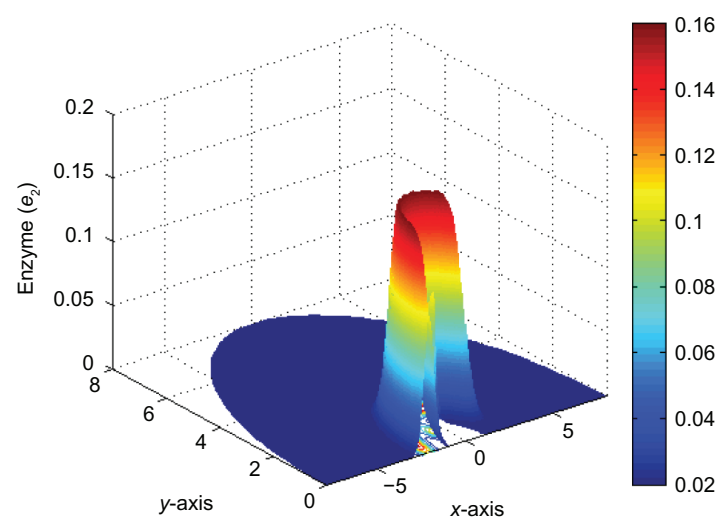

Figure S2 Snapshots of evolution of specific enzyme type II ( $\left.\mathrm{e}_{2}\right)$ for $\left.\left.\left.\mathbf{A}\right) t=\mathrm{I}, \mathbf{B}\right) t=5, \mathbf{C}\right) t=15$, and $\left.\mathbf{D}\right) t=30$ days. The propagation was initiated from periodical releases of one microphage per 0.5 day, and the period was 0.5 day. Release locations were equally distributed on the outer boundary of the wound. 
A

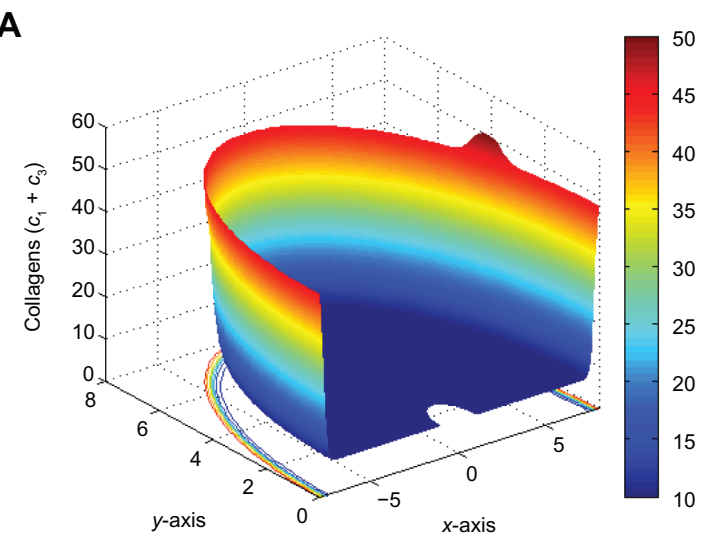

C

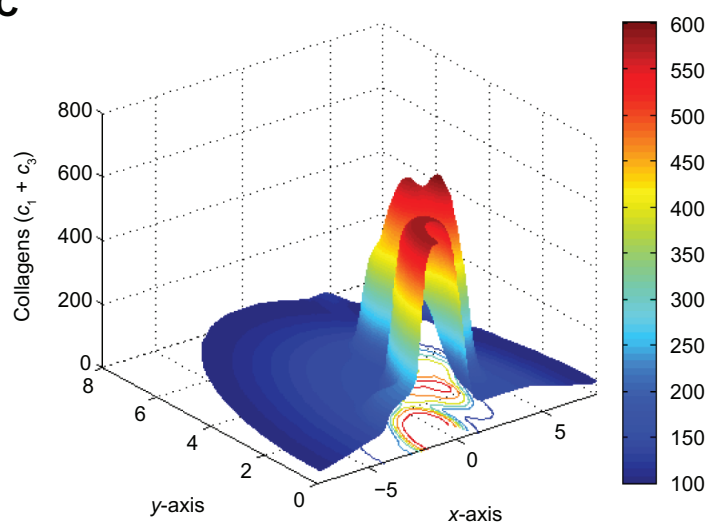

B

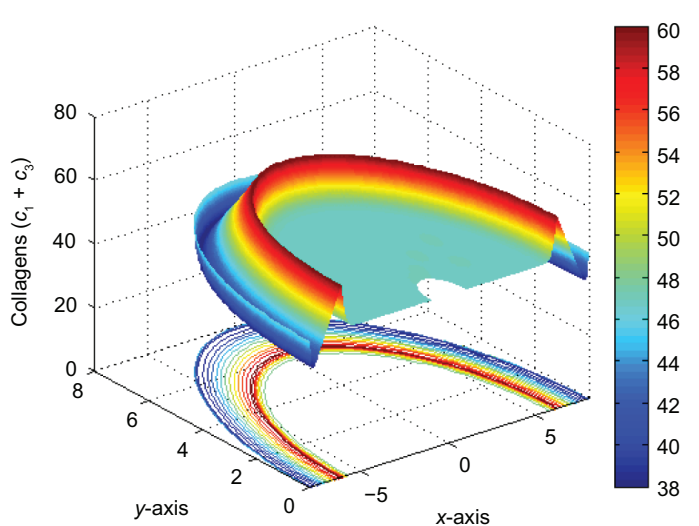

D

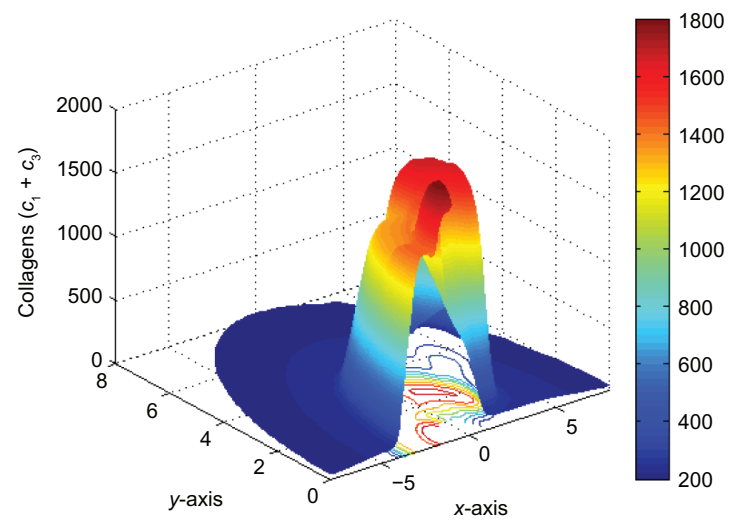

Figure S3 Snapshots of evolution of the total collagen production $c_{1}+c_{3}$ for A) $\left.\left.t=1, \mathbf{B}\right) t=5, \mathbf{C}\right) t=15$, and $\left.\mathbf{D}\right) t=30$ days following the release of one macrophage per 0.5 day.

Open Access Bioinformatics

Dovepress

\section{Publish your work in this journal}

Open Access Bioinformatics is an international, peer-reviewed, open access journal publishing original research, reports, reviews and commentaries on all areas of bioinformatics. The manuscript management system is completely online and includes a very quick and fair peer-review system. Visit http://www.dovepress.com/testimonials.php to read real quotes from published authors. 